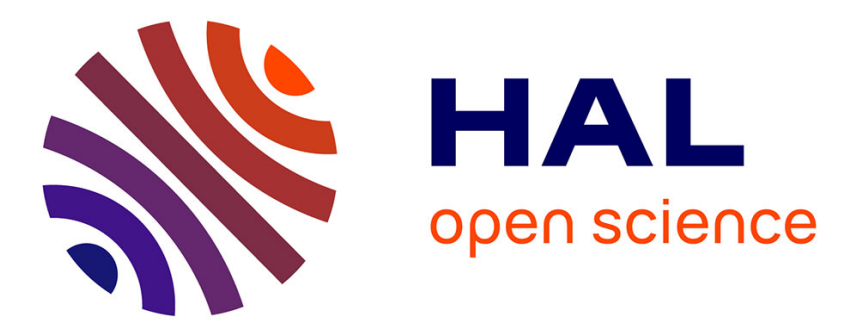

\title{
Efficient numerical computations of yield stress fluid flows using second-order cone programming
}

\author{
Jeremy Bleyer, Mathilde Maillard, Patrick de Buhan, Philippe Coussot
}

\section{To cite this version:}

Jeremy Bleyer, Mathilde Maillard, Patrick de Buhan, Philippe Coussot. Efficient numerical computations of yield stress fluid flows using second-order cone programming. Computer Methods in Applied Mechanics and Engineering, 2015, 283, pp.599 - 614. 10.1016/j.cma.2014.10.008 . hal-01081508

\section{HAL Id: hal-01081508 https://hal.science/hal-01081508}

Submitted on 8 Nov 2014

HAL is a multi-disciplinary open access archive for the deposit and dissemination of scientific research documents, whether they are published or not. The documents may come from teaching and research institutions in France or abroad, or from public or private research centers.
L'archive ouverte pluridisciplinaire HAL, est destinée au dépôt et à la diffusion de documents scientifiques de niveau recherche, publiés ou non, émanant des établissements d'enseignement et de recherche français ou étrangers, des laboratoires publics ou privés. 


\title{
Efficient numerical computations of yield stress fluid flows using second-order cone programming
}

\author{
Jeremy Bleyer ${ }^{\mathrm{a}, *}$, Mathilde Maillard ${ }^{\mathrm{a}}$, Patrick de Buhan ${ }^{\mathrm{a}}$, Philippe Coussot $^{\mathrm{a}}$ \\ ${ }^{a}$ Université Paris-Est, Laboratoire Navier, \\ Ecole des Ponts ParisTech-IFSTTAR-CNRS (UMR 8205) \\ 6-8 av Blaise Pascal, Cité Descartes, 77455 Champs-sur-Marne, FRANCE
}

\begin{abstract}
This work addresses the numerical computation of the two-dimensional flow of yield stress fluids (with Bingham and Herschel-Bulkley models) based on a variational approach and a finite element discretization. The main goal of this paper is to propose an alternative optimization method to existing procedures such as penalization and augmented Lagrangian techniques. It is shown that the minimum principle for Bingham and Herschel-Bulkley yield stress fluid steady flows can, indeed, be formulated as a second-order cone programming (SOCP) problem, for which very efficient primal-dual interior point solvers are available. In particular, the formulation does not require any regularization of the visco-plastic model as is usually the case for existing techniques, avoiding therefore the difficult choice of the regularization parameter. Besides, it is also unnecessary to adopt a mixed stress-velocity approach or discretize explicitly auxiliary variables as frequently proposed in existing methods. Finally, the performance of dedicated SOCP solvers, like the MOSEK software package, enables to solve large-scale problems on a personal computer within seconds only. The proposed method will be validated on classical benchmark examples and used to simulate the flow generated around a plate during its withdrawal from a bath of yield stress fluid.
\end{abstract}

Keywords: yield stress fluids, viscoplasticity, Bingham model, Herschel-Bulkley model, finite element method, second-order cone programming

\section{Introduction}

Yield stress fluids are encountered in a wide range of applications: toothpastes, cement, mortar, foams, muds, mayonnaise, etc. The fundamental character of these fluids is that they are able to flow (i.e. deform indefinitely) only if they are submitted to a stress larger than a critical value, otherwise they deform in a finite way like solids. Here, we will focus

\footnotetext{
${ }^{*}$ Correspondence to: J. Bleyer, Laboratoire Navier, 6-8 av Blaise Pascal, Cité Descartes, 77455 Champssur-Marne, France, Tel : +33 (0)1 64153659

Email address: jeremy.bleyer@enpc.fr (Jeremy Bleyer)

$U R L:$ https://sites.google.com/site/bleyerjeremy/ (Jeremy Bleyer)
} 
on the flow characteristics of non-thixotropic yield stress fluids which can, as a good first approximation, be described as simple yield stress fluids, i.e. for which the yield stress and more generally the apparent viscosity are independent of the flow history. Even in that case the flow characteristics of such materials are difficult to predict as they involve permanent or transient solid and liquid regions whose location cannot generally be determined a priori. Uniform flow of these fluids in simple geometries can easily be described analytically. However there exists a wide set of more complex situations for which analytical description remains strongly approximate or even impossible, so that numerical simulations only can provide useful information or predictions. This concerns stationary flows in channels of varying cross-section such as extrusion, expansion, flow through porous medium, or transient flows such as flows around obstacles, spreading, spin-coating, squeeze flow, elongation, etc.

Various numerical methods have been proposed to simulate the flow of visco-plastic fluids. The main difficulty arising in such simulations is the non-regular constitutive relation between stresses and strain rates which distinguish between a rigid solid domain if the stress state is below the yield stress and a liquid flow otherwise. One major approach consists in regularizing the constitutive behavior by introducing an artificial parameter (which can be viewed as a high viscosity below yield stress e.g. bi-viscous model) to obtain a smoother problem [1-4]. However, the distinction between solid and liquid regions is then much harder to detect and the solution process can deteriorate when changing the regularization parameter. The other main approach avoids any regularization and aims at solving the associated non-smooth variational problem with different optimization techniques, the main example being the Augmented Lagrangian approach [5-7]. Mesh adaptive strategies have also been proposed to enhance the prediction of the fluid-solid boundaries [6]. An interesting review of such techniques can be found in [8].

The simulation of yield stress fluid flows is actually strongly related to the limit analysis (or yield design in a more general manner) of mechanical structures which aims at computing the limit loads and associated failure mechanisms of rigid perfectly-plastic structures $[9,10]$. More precisely, when viscous effects are dominated by yield effects $(B i \rightarrow \infty)$ both problems become coincident. In the past decades, computational limit analysis also received an important attention, especially due to the development of mathematical programming tools used to solve the arising optimization problems. Interestingly, regularized models have also first been proposed for limit analysis problems [11, 12], leading to the same difficulties as those previously mentioned. Linear programming solvers have then been proposed in the case of piecewise linear yield surfaces [13-16]. This approach presented the advantage of avoiding any regularization parameter and enabled to solve a linear optimization problem quite efficiently for medium scale problems. However, its computational efficiency was limited by the important number of additional constraints introduced by the linearization of the non-linear yield surface. Efficient algorithms, namely interior point solvers, have then been developed for linear problems. Their extension to non-linear optimization problems and especially second-order cone programming (SOCP) resulted in an important breakthrough as regards the development of computational capabilities for limit analysis [17-19]. These algorithms have also been implemented in commercial codes such as the MosEK software 
package [20]. Remarkably, a large number of traditional yield surfaces can be expressed using conic constraints so that limit analysis problems can be formulated within SOCP [21]. Most of the recent publications in the field of limit analysis are now considering such approaches as the most efficient ones and apply them to a large number of problems : 2D plane strain problems [22, 23], frame structures [24], thin plates in bending [25, 26] ..

The aim of this paper is then to show how such methods, successfully used in the limit analysis of mechanical structures, can be transposed to the simulation of yield stress fluids. In particular, it will be shown that it is possible to take both Bingham and Herschel-Bulkley models into account and that the computational performances are very interesting as compared to previously mentioned standard techniques. Section 2 is devoted to the variational formulation of visco-plastic flows. Section 3 will deal with the finite element discretization. The formulation of the discrete problem as a second-order cone program, which is the main novelty of this paper, is considered in section 4 . Finally, section 5 will present different illustrative examples used to validate the procedure by comparing numerical solutions to analytical ones and experimental measures, as well as to assess its numerical efficiency.

\section{Minimum principle for yield stress fluids}

\subsection{Governing equations}

Let $\Omega$ denote the two-dimensional fluid domain, $\underline{u}=u_{x} \underline{e}_{x}+u_{y} \underline{e}_{y}$ be the fluid velocity field and $\underline{\underline{\sigma}}$ the stress tensor field. The creeping flow of an incompressible yield stress fluid (characterized by a yield shear stress $\tau_{0}$ ) is described by the following set of equations and boundary conditions :

$$
\begin{aligned}
& \operatorname{div} \underline{\underline{\sigma}}+\underline{f}=\underline{0} \\
& \operatorname{div} \underline{u}=0 \\
& \underline{\underline{d}}=\frac{1}{2}\left(\underline{\underline{\nabla u}}+{ }^{T} \underline{\underline{\nabla u}}\right) \\
& \begin{cases}\underline{\underline{s}}=2 \mu \underline{\underline{d}}+2 \tau_{0} \frac{\underline{\underline{d}}}{\|\underline{\underline{d}}\|} & \text { if } \sqrt{\frac{1}{2} \underline{\underline{s}}: \underline{\underline{s}}} \geq \tau_{0} \\
\underline{\underline{d}}=0 & \text { if } \sqrt{\frac{1}{2} \underline{\underline{s}}: \underline{\underline{s}}}<\tau_{0}\end{cases} \\
& \underline{u}=\underline{U}^{d}
\end{aligned}
$$

where $\underline{\underline{d}}$ is the strain rate tensor, $\underline{f}$ is the volume body force density, $\underline{U}^{d}$ the prescribed velocity, $\underline{\underline{s}}=\underline{\underline{\sigma}}-\frac{1}{3}(\operatorname{tr} \underline{\underline{\sigma}}) \underline{\underline{1}}$ is the deviatoric stress tensor and where $\|\underline{\underline{d}}\|=\sqrt{2 \underline{\underline{d}}: \underline{\underline{d}}}$. Equation (4) in particular represents the constitutive equation which distinguishes a rigid region $\left(\sqrt{\frac{1}{2} \underline{\underline{s}}: \underline{\underline{s}}}<\tau_{0}\right)$ from a yielded region $\left(\sqrt{\frac{1}{2} \underline{\underline{s}}: \underline{\underline{s}}} \geq \tau_{0}\right)$. Note that the constitutive equation can also be written as $\underline{\underline{d}}=\frac{\underline{\underline{s}}}{2 \mu} \sup \left\{0 ;\left(1-\frac{\tau_{0}}{\sqrt{\frac{1}{2} \underline{\underline{s}}: \underline{\underline{s}}}}\right)\right\}$.

In this context, the Bingham model assumes that the viscosity $\mu$ is a constant, whereas more 
sophisticated models assume that it actually depends on the strain rate tensor. This is the case of the Herschel-Bulkley (HB) model which assumes the following power-law relationship :

$$
\mu=\mu(\underline{\underline{d}})=K\|\underline{\underline{d}}\|^{m-1}
$$

characterized by the power-law exponent $m>0$ and the consistency $K$. Since the Bingham model is a particular case $(m=1)$ of the HB model, we will work from now on with the HB model.

\subsection{Minimum principle}

It can be shown [27] that the velocity field $\underline{u}$ solution of the above system of equations is the optimal point of the following minimization problem :

$$
\underline{u}=\arg \min _{\underline{v} \in \mathcal{V}} \frac{1}{m+1} \int_{\Omega} \mu(\underline{\underline{d}}) \underline{\underline{d}}: \underline{\underline{d}} d \Omega+\int_{\Omega} 2 \tau_{0} \frac{\underline{\underline{d}}}{\|\underline{\underline{d}}\|}: \underline{\underline{d}} d \Omega-\int_{\Omega} \underline{f} \cdot \underline{v} d \Omega
$$

which simplifies into :

$$
\underline{u}=\arg \min _{\underline{v} \in \mathcal{V}} \frac{1}{m+1} \int_{\Omega} K\|\underline{\underline{d}}\|^{m+1} d \Omega+\int_{\Omega} \tau_{0}\|\underline{\underline{d}}\| d \Omega-\int_{\Omega} \underline{f} \cdot \underline{v} d \Omega
$$

where $\underline{\underline{d}}$ is the strain rate attached to the minimization variable velocity field $\underline{v}$ and $\mathcal{V}=$ $\left\{\underline{v} \mid \operatorname{div} \underline{v}=0\right.$ in $\Omega, \underline{v}=\underline{U}^{d}$ on $\left.\partial \Omega\right\}$ is the set of incompressible and kinematically admissible velocity fields, i.e. which are continuous, differentiable and satisfy the boundary conditions. Note that, in the case when inertial effects are not neglected, the solution at a given time can be obtained by solving a similar formulation involving the solution at the preceding time step and a quadratic term with respect to the velocity field.

\subsection{Existing techniques}

One can remark that the objective functional to be minimized is non-linear and nondifferentiable due to the yield stress term in $\|\underline{d}\|$, the derivative of which is not defined if $\underline{\underline{d}}=\underline{\underline{0}}$. This term makes it impossible to use standard gradient descent algorithms to perform the minimization, thus leading to the development of many regularized models to smooth the functional.

The augmented Lagrangian approach has been proposed to tackle this issue without regularizing the non-smooth term by uncoupling non-linearities and derivatives. To this end, an auxiliary variable $\underline{\gamma}$ is introduced and forced to be equal to the velocity gradient by penalization, using a Lagrange multiplier $\underline{\underline{\tau}}$ having the dimension of a stress. The following augmented Lagrangian is defined :

$$
\begin{aligned}
& \mathcal{L}_{r}(\underline{v}, \underline{\underline{\gamma}} ; \underline{\underline{\tau}}, \underline{p})= \frac{1}{m+1} \int_{\Omega} K\left\|\underline{\underline{\gamma^{s}}}\right\|^{m+1} d \Omega+\int_{\Omega} \tau_{0}\left\|\underline{\underline{\gamma}}^{s}\right\| d \Omega-\int_{\Omega} \underline{f} \cdot \underline{v} d \Omega \\
&+\int_{\Omega} p \cdot \operatorname{div} \underline{u} d \Omega+\int_{\Omega}(\underline{\underline{\gamma}}-\underline{\underline{\nabla v}}): \underline{\underline{\tau}} d \Omega+\frac{r}{2} \int_{\Omega}(\underline{\underline{\gamma}}-\underline{\underline{\nabla v}})^{2} d \Omega \\
& 4
\end{aligned}
$$


where $r$ is a strictly positive penalization parameter, $\underline{\underline{\gamma}}^{s}$ is the symmetric part of $\underline{\underline{\gamma}}$ and $p$ is the Lagrange multiplier of the incompressibility (no volume change) condition, having the dimension of a pressure. The interest of this method is that the saddle points of this Lagrangian correspond to the solution $\underline{u}$ and $\underline{\underline{\gamma}}=\underline{\underline{\nabla u}}$ at the optimum. Besides, the resolution of the augmented saddle point problem is easier than the initial one because the Lagrangian is a quadratic function of the velocity field and can, therefore, be easily minimized with respect to $\underline{v}$ for fixed $\underline{\underline{\gamma}}, p$ and $\underline{\underline{\tau}}$. In the end, an iterative procedure involving simple minimization sub-problems can be derived.

Despite being certainly the most efficient approach available so far to tackle such a problem, four variable fields $\underline{v}, \underline{\underline{\gamma}}, p$ and $\underline{\underline{\tau}}$ must be discretized to solve the problem and special attention must be paid on the choice of the discretization spaces. Another important point as regards the practical use of the Augmented Lagrangian, is that the choice of an optimal value for the penalization parameter $r$ can be a critical issue [8, 27] and, most certainly, problem-dependent. Besides, other numerical parameters involved in the iterative actualization of the mechanical fields can influence the convergence rate of the algorithm so that an optimal choice of their values remains an open question [7]. Finally, despite the large number of papers using Augmented Lagrangian approaches of visco-plastic fluids, very few of them mention the computing time needed to minimize the functional, which may be quite important and thus prohibitive, to the authors opinion, for large-scale problems.

\section{Finite element discretization}

In this section, the finite element discretization of the minimum principle (6) will be considered. In particular, only the velocity field $\underline{v}$ will be discretized and matrices expressing the strain rate-velocity compatibility equation and the divergence-free condition will be written.

As already mentioned, problem (6) is quite similar to those arising in the upper bound kinematical approach of plane strain limit analysis problems with a von Mises strength criterion, the only difference arising from the viscosity term which is not present in limit analysis. It will be seen in the next section that this supplementary term can be treated quite easily using second-order cone programming formulations. Hence, the finite element discretization adopted in this section takes full advantage of the work done in the field of limit analysis. In particular, various works have shown that a quadratic interpolation of continuous $^{1}$ velocity fields must be considered in order to avoid volumetric locking problems caused by the incompressibility condition.

\footnotetext{
${ }^{1}$ Contrary to limit analysis, which corresponds to a vanishing viscosity, the kinematically admissible velocity fields must remain continuous.
} 


\subsection{Formulation of the discretized minimum principle}

The fluid domain $\Omega$ is supposed to be discretized into $N_{E}$ 6-noded triangular finite elements (it will supposed, to simplify that the triangle edges are straight). The velocity field inside a given element $e$ is interpolated quadratically by its values $\underline{v}^{i}$ at the six nodes (three vertices and three mid-side nodes) :

$$
\underline{v}^{(e)}(x, y)=\sum_{i=1}^{6} N_{i}(\xi, \eta) \underline{v}^{i}
$$

where $(\xi, \eta)$ are the coordinates in a reference triangle and $N_{i}$ are the 6 quadratic shape functions. Let $\mathbf{J}_{e}$ be the jacobian matrix of the transformation from the current element to the reference triangle, then the strain rate is expressed inside this element as follows :

$$
\tilde{\mathbf{d}}^{e}(x, y)=\left\{\begin{array}{c}
d_{x x} \\
d_{y y} \\
2 d_{x y}
\end{array}\right\}=\mathbf{J}_{e}^{-T} \underbrace{\left[\begin{array}{ccccccc}
N_{1, \xi} & 0 & N_{2, \xi} & 0 & \ldots & N_{6, \xi} & 0 \\
0 & N_{1, \eta} & 0 & N_{2, \eta} & \ldots & 0 & N_{6, \eta} \\
N_{1, \eta} & N_{1, \xi} & N_{2, \eta} & N_{2, \xi} & \ldots & N_{6, \eta} & N_{6, \xi}
\end{array}\right]}_{\mathbf{D N}(\xi, \eta)} \mathbf{v}^{e}
$$

where $\mathbf{v}^{e}=\left\langle\begin{array}{llllll}v_{x}^{1} & v_{y}^{1} & v_{x}^{2} & \ldots & v_{y}^{6}\end{array}\right\rangle^{T}$ collects the twelve nodal degrees of freedom of element $e$. The strain rate will now be expressed at $n g$ Gauss points inside the triangle so that we have :

$$
\mathbf{d}^{e}=\left\{\begin{array}{c}
\tilde{\mathbf{d}}^{e}\left(x_{1}, y_{1}\right) \\
\vdots \\
\tilde{\mathbf{d}}^{e}\left(x_{n g}, y_{n g}\right)
\end{array}\right\}=\left[\begin{array}{c}
\mathbf{J}_{e}^{-T} \mathbf{D N}\left(\xi_{1}, \eta_{1}\right) \\
\vdots \\
\mathbf{J}_{e}^{-T} \mathbf{D N}\left(\xi_{n g}, \eta_{n g}\right)
\end{array}\right] \mathbf{v}^{e}
$$

The objective function $\mathcal{J}(\underline{v})$ in (6) is then computed by summing over all elements $\Omega_{e}$ :

$$
\begin{aligned}
\mathcal{J}(\underline{v}) & =\sum_{e=1}^{N_{E}}\left(\frac{1}{m+1} \int_{\Omega_{e}} K\|\underline{\underline{d}}\|^{m+1} d \Omega+\int_{\Omega_{e}} \tau_{0}\|\underline{\underline{d}}\| d \Omega-\int_{\Omega_{e}} \underline{f} \cdot \underline{v} d \Omega\right) \\
& =\sum_{e=1}^{N_{E}}\left(\frac{K_{e}}{m+1} \int_{\Omega_{e}}\left\|\mathbf{Q} \tilde{\mathbf{d}}^{e}\right\|^{m+1} d \Omega+\tau_{0, e} \int_{\Omega_{e}}\left\|\mathbf{Q} \tilde{\mathbf{d}}^{e}\right\| d \Omega\right)-\mathbf{f}^{T} \cdot \mathbf{v}
\end{aligned}
$$

where $K$ and $\tau_{0}$ are assumed to be piecewise constant on each element (of values $K_{e}$ and $\left.\tau_{0, e}\right), \mathbf{f}$ is the equivalent nodal force vector, $\mathbf{v}$ of length $N_{v}$ collects the nodal values of the velocity field and $\mathbf{Q}=\left[\begin{array}{lll}2 & 0 & 0 \\ 0 & 2 & 0 \\ 0 & 0 & 1\end{array}\right]$ (the norm being here the traditional Euclidean norm for vectors). Finally, both elementary integrals are approximated using a Gaussian quadrature on the reference triangle with weights $\omega_{g}$ for $g=1, \ldots, n g$ :

$$
\mathcal{J}(\underline{v}) \approx \sum_{e=1}^{N_{E}} \sum_{g=1}^{n g} \omega_{g} \operatorname{det} \mathbf{J}_{e}\left(\frac{K_{e}}{m+1}\left\|\mathbf{Q} \tilde{\mathbf{d}}_{g}^{e}\right\|^{m+1}+\tau_{0, e}\left\|\mathbf{Q} \tilde{\mathbf{d}}_{g}^{e}\right\|\right)-\mathbf{f}^{T} \cdot \mathbf{v}
$$


Finally, the discretized minimization problem can be written as :

$$
\begin{array}{ll}
\min & \sum_{e=1}^{N_{E}} \sum_{g=1}^{n g} \omega_{g} \operatorname{det} \mathbf{J}_{e}\left(\frac{K_{e}}{m+1}\left\|\mathbf{Q} \tilde{\mathbf{d}}_{g}^{e}\right\|^{m+1}+\tau_{0, e}\left\|\mathbf{Q} \tilde{\mathbf{d}}_{g}^{e}\right\|\right)-\mathbf{f}^{T} \cdot \mathbf{v} \\
\text { s.t. } & \tilde{\mathbf{d}}_{g}^{e}=\mathbf{J}_{e}^{-T} \mathbf{D N}_{g} \mathbf{v}^{e} \quad \forall g=1, \ldots, n g \quad \forall e=1, \ldots, N_{E} \\
& \langle 110\rangle \cdot \tilde{\mathbf{d}}_{g}^{e}=0 \\
& \mathbf{v}_{I}=\mathbf{U}^{d} \\
& \forall I \in B C
\end{array}
$$

where the second constraint expresses the divergence-free condition and the third constraint fixes the values of $\underline{v}$ on the boundary to the prescribed value $\underline{U}^{d}, I$ is spanning the set $B C$ of all indexes corresponding to a prescribed component of $\underline{v}$.

Written in this form, problem (7) is the discrete version of (6) and all the remarks concerning the difficulty of the corresponding minimization problem are still present. The next section is, thus, devoted to the reformulation of this problem as second-order cone programs.

\subsection{Treatment of axisymmetric problems}

Let us just mention here that the treatment of axisymmetric problems about an axis $z$ is very similar to that of $2 \mathrm{D}$ plane strain problems. Indeed, in this case, the velocity vector is $\underline{u}=u_{r}(r, z) \underline{e}_{r}+u_{z}(r, z) \underline{e}_{z}$ which is discretized in the same way as in the 2D case. The strain tensor possesses here 4 non-zero components so that we have :

$$
\tilde{\mathbf{d}}^{e}(r, z)=\left\{\begin{array}{c}
d_{r r} \\
d_{\theta \theta} \\
d_{z z} \\
2 d_{x y}
\end{array}\right\}=\mathbf{J}_{e}^{-T} \underbrace{\left[\begin{array}{ccccccc}
N_{1, \xi} & 0 & N_{2, \xi} & 0 & \ldots & N_{6, \xi} & 0 \\
N_{1} / r & 0 & N_{2} / r & 0 & \ldots & N_{6} / r & 0 \\
0 & N_{1, \eta} & 0 & N_{2, \eta} & \ldots & 0 & N_{6, \eta} \\
N_{1, \eta} & N_{1, \xi} & N_{2, \eta} & N_{2, \xi} & \ldots & N_{6, \eta} & N_{6, \xi}
\end{array}\right]}_{\mathbf{D N}(\xi, \eta)} \mathbf{v}^{e}
$$

we also have now $\mathbf{Q}=\left[\begin{array}{llll}2 & 0 & 0 & 0 \\ 0 & 2 & 0 & 0 \\ 0 & 0 & 2 & 0 \\ 0 & 0 & 0 & 1\end{array}\right]$ and $\left\langle\begin{array}{lllll}1 & 1 & 1 & 0\rangle\end{array} \cdot \tilde{\mathbf{d}}_{g}^{e}=0\right.$ for the divergence-free condition.

Finally, all elementary integral quadratures are modified to take the $r d r$ term into account (the $2 \pi$ coefficient is simplified).

\section{Second-order cone programming formulation}

\subsection{Standard second-order cone programs}

Second-order cone programming (SOCP) is a particular class of convex programming which consists of the minimization/maximization of a linear function of the optimization variable vector $\mathbf{x} \in \mathbb{R}^{n}$, under linear equality and/or inequality constraints and particular 
non-linear constraints, namely second-order cone (SOC) constraints. Hence, in the case when there are no linear inequality constraints, a SOCP problem can be written as :

$$
\begin{array}{cl}
\min _{\mathbf{x}} & \mathbf{c}^{T} \cdot \mathbf{x} \\
\text { s.t. } & \mathbf{A x}=\mathbf{b} \\
& \mathbf{x} \in \mathcal{K}
\end{array}
$$

where $\mathbf{A} \in \mathbb{R}^{m \times n}, \mathbf{b} \in \mathbb{R}^{m}, \mathbf{c} \in \mathbb{R}^{n}$ and $\mathcal{K}$ is a second-order cone (or a tensorial product of second-order cones $\mathcal{K}=\mathcal{K}_{1} \times \mathcal{K}_{2} \times \ldots \times \mathcal{K}_{s}$ ). Typical examples of second-order cones of dimension $p \geq 3$ are the following :

- the positive orthant :

$$
\mathcal{K}=\mathbb{R}^{p+}=\left\{\mathbf{y} \in \mathbb{R}^{p} \mid y_{i} \geq 0 \quad \forall i=1, \ldots, p\right\}
$$

- the Lorentz cone (or "ice-cream" cone) :

$$
\mathcal{K}=\mathcal{L}_{p}=\left\{\mathbf{y} \in \mathbb{R}^{p} \mid y_{1} \geq \sqrt{y_{2}^{2}+\ldots+y_{p}^{2}}\right\}
$$

- the rotated Lorentz cone :

$$
\mathcal{K}=\mathcal{R} \mathcal{L}_{p}=\left\{\mathbf{y} \in \mathbb{R}^{p} \mid 2 y_{1} y_{2} \geq y_{3}^{2}+\ldots+y_{p}^{2}\right\}
$$

The first example is a particular case. It is in fact a polyhedral cone since it can be expressed using linear inequalities. On the contrary, the last two examples are expressed using non-linear constraints (involving euclidean norms) and cannot be expressed using linear constraints only.

In the particular case when (8) contains only polyhedral cones, SOCP reduces to linear programming (LP) in which objective function, equality and inequality constraints are all linear. Efficient algorithms dedicated to solve LP problems have received considerable attention and efficient primal-dual interior point solvers are now available and able to solve large-scale (up to millions of unkowns) problems within very reasonable computing times. Those algorithms have been extended to the case of SOCP problems since, both problems are quite close despite the presence of non-linearities. Dedicated SOCP solvers like the MOSEK software package are available and proved to be almost as efficient as in linear programming. Hence, MoseK can solve problems of the form (8) with cones expressed as tensorial products of Lorentz or rotated Lorentz cones. It is quite interesting to remark that a large variety of non-linear optimization problems can actually be reformulated as SOCP problems.

\subsection{Treatment of the yield stress term}

Comparing (7) to (8), it can be seen that they both involve linear equality constraints, but the objective function of (7) is nonlinear due to the viscosity and yield stress terms. Let us first focus on the yield stress term and forget for now the viscosity term. Following 
the procedure proposed in [17] for minimizing a sum of Euclidean norms, $n g \cdot N_{E}$ auxiliary variables $\mathbf{r}_{g}^{e} \in \mathbb{R}^{3}$ and $t_{g}^{e}$ are introduced as follows :

$$
\begin{aligned}
& \min \left(\text { viscosity term) }+\sum_{e=1}^{N_{E}} \sum_{g=1}^{n g} \omega_{g} \operatorname{det} \mathbf{J}_{e} \tau_{0, e} t_{g}^{e}-\mathbf{f}^{T} \cdot \mathbf{v}\right. \\
& \text { s.t. }\left\|\mathbf{r}_{g}^{e}\right\| \leq t_{g}^{e} \quad \forall g=1, \ldots, n g \quad \forall e=1, \ldots, N_{E} \\
& \mathbf{r}_{g}^{e}=\mathbf{Q} \mathbf{J}_{e}^{-T} \mathbf{D N} \mathbf{N}_{g} \mathbf{v}^{e} \\
& \left\langle\begin{array}{lll}
1 / 2 & 1 / 2 & 0\rangle \cdot \mathbf{r}_{g}^{e}=0
\end{array}\right. \\
& \mathbf{v}_{I}=\mathbf{U}^{d} \quad \forall I \in B C
\end{aligned}
$$

Now, it can be seen that the objective function is a linear function of the auxiliary variables $t_{g}^{e}$, vectors $\mathbf{r}_{g}^{e}$ correspond to the strain variables $\tilde{\mathbf{d}}_{g}^{e}$ with matrix $\mathbf{Q}$ as a factor and $n g \cdot N_{E}$ constraints of the form $\|\mathbf{r}\| \leq t$ have been added. These constraints are no more than a Lorentz cone constraint on a 4 -dimensional vector $(t, \mathbf{r})$. An important remark is that since the objective function is minimized and that the coefficients $\omega_{g} \operatorname{det} \mathbf{J}_{e} \tau_{0, e}$ are positive, the solution of the problem will be obtained when all auxiliary variables $t$ will saturate the conic constraint $\|\mathbf{r}\| \leq t$ such that, at the optimum, $t=\|\mathbf{r}\|$. In the end, problems (9) and (7) (without the viscosity) are perfectly equivalent. Besides, introducing the vector of unknowns

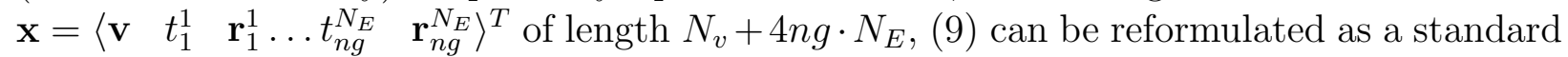
SOCP problem :

$$
\begin{aligned}
& \min \left\langle-\mathbf{f} \quad \mathbf{c}_{y}\right\rangle \cdot \mathbf{x} \\
& \text { s.t. }\left[\begin{array}{cc}
\mathbf{B} & -\mathbf{I} \\
\mathbf{0} & \mathbf{T} \\
\mathbf{L}_{I} & \mathbf{0}
\end{array}\right] \mathbf{x}=\left\{\begin{array}{c}
\mathbf{0} \\
\mathbf{0} \\
\mathbf{U}^{d}
\end{array}\right\} \\
& \mathbf{x} \in \mathbb{R}^{N_{v}} \times\left(\mathcal{L}_{4}\right)^{n g \cdot N_{E}}
\end{aligned}
$$

where $\mathbf{c}_{y}=\left\langle\begin{array}{lllllllll}\omega_{1} \operatorname{det} \mathbf{J}_{1} \tau_{0,1} & 0 & 0 & 0 & \ldots & \omega_{n g} \operatorname{det} \mathbf{J}_{N_{E}} \tau_{0, N_{E}} & 0 & 0 & 0\end{array}\right\rangle$, B corresponds to matrices $\mathbf{Q} \mathbf{J}_{e}^{-T} \mathbf{D} \mathbf{N}_{g}$ assembled at the global level, $\mathbf{L}_{I}$ is a matrix which is used to collect only the components $I \in B C$ of $\mathbf{v}$ and

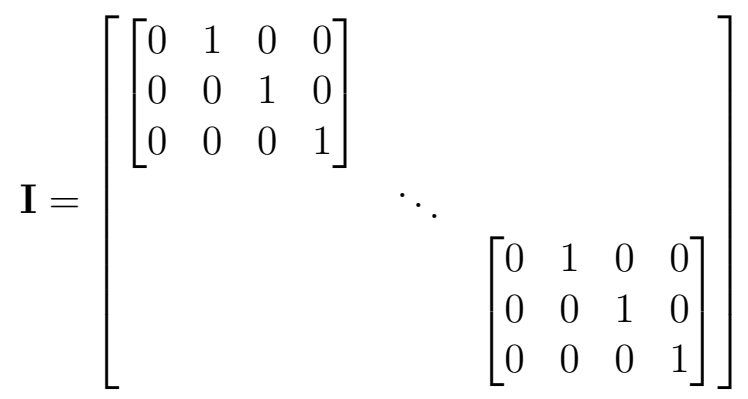

$$
\begin{aligned}
& \mathbf{T}=\left[\begin{array}{lllllllll}
{\left[\begin{array}{llllll}
0 & 1 / 2 & 1 / 2 & 0
\end{array}\right]} & & & & \\
& & & & \ddots & & & & \\
& & & & & {\left[\begin{array}{lllll}
0 & 1 / 2 & 1 / 2 & 0
\end{array}\right]}
\end{array}\right]
\end{aligned}
$$




\subsection{Treatment of the viscosity term for a Bingham model}

It has been seen how to treat the yield stress term in the objective function using SOC constraints. Now, let us focus on the viscosity term in the case of a Bingham model, i.e. $m=1$. The viscosity term is here a quadratic function of the components of the strain rate tensor since $\|\mathbf{Q} \tilde{\mathbf{d}}\|^{2}=2 d_{x x}^{2}+2 d_{y y}^{2}+4 d_{x y}^{2}$. This term can, once again, be efficiently replaced by a conic constraint by introducing two supplementary auxiliary variables $s$ and $w$. Once again, the goal is to replace the objective by, let say, $s$ and introduce a conic constraint such that $s=\|\mathbf{Q} \tilde{\mathbf{d}}\|^{2}=\|\mathbf{r}\|^{2}$ at the optimum. It can be seen that adding the following constraints $[20]:$

$$
\begin{gathered}
2 w s \geq\|\mathbf{r}\|^{2} \\
w=1 / 2
\end{gathered}
$$

will enforce $s=\|\mathbf{r}\|^{2}$ at the optimum using a rotated Lorentz cone on the 5-dimensional vector $(w, s, \mathbf{r})$. The initial problem $(7)$ can then be reformulated as follows:

$$
\begin{array}{ll}
\min & \sum_{e=1}^{N_{E}} \sum_{g=1}^{n g} \omega_{g} \operatorname{det} \mathbf{J}_{e}\left(\frac{K_{e}}{2} s_{g}^{e}+\tau_{0, e} t_{g}^{e}\right)-\mathbf{f}^{T} \cdot \mathbf{v} \\
\text { s.t. } & \left\|\mathbf{r}_{g}^{e}\right\| \leq t_{g}^{e} \quad \forall g=1, \ldots, n g \quad \forall e=1, \ldots, N_{E} \\
& \left\|\mathbf{r}_{g}^{e}\right\|^{2} \leq 2 w s_{g}^{e} \\
& w=1 / 2 \\
& \mathbf{r}_{g}^{e}=\mathbf{Q} \mathbf{J}_{e}^{-T} \mathbf{D} \mathbf{N}_{g} \mathbf{v}^{e} \\
& \langle 1 / 21 / 20\rangle \cdot \mathbf{r}_{g}^{e}=0 \quad \\
& \mathbf{v}_{I}=\mathbf{U}^{d} \quad \forall I \in B C
\end{array}
$$

which once again can be cast as a standard SOCP problem since the objective function is linear and all constraints are either linear, a Lorentz cone or a rotated Lorentz cone.

\subsection{Treatment of the viscosity term for a Herschel-Bulkley model}

Finally, the case of the Herschel-Bulkley model is slightly more complicated but can still be written using SOC constraints in general. Indeed, following the same line of reasoning as before, one would like to express a constraint of the form $\|\mathbf{r}\|^{m+1} \leq s$ when $m \neq 1$ and $m>0$ using SOC constraints. Power-law constraints can indeed be expressed as such if the exponent $m+1$ is rational, i.e. of the form $m+1=p / q$ with $p, q \in \mathbb{N}^{*}$. The general procedure to do so follows results from [28] and is described in the MosEK documentation [20]. For the sake of simplicity, we will only present an example of the procedure in the case when $m=0.4$ i.e. $m+1=1.4=7 / 5$. Observing that :

$$
\|\mathbf{r}\|^{7 / 5} \leq s \Longleftrightarrow \begin{aligned}
& \|\mathbf{r}\| \leq x \\
& x^{8} \leq x s^{5}
\end{aligned}
$$

the last constraint is equivalent to :

$$
x^{8} \leq 2^{12} y_{1} \cdots y_{8}, \quad y_{1}=x, y_{2}=\cdots=y_{6}=s, y_{7}=1, y_{8}=2^{-12}
$$


Now, introducing auxiliary variables and 3 levels of rotated cones, $x^{8} \leq 2^{12} y_{1} \cdots y_{8}$ is equivalent to :

$$
\begin{gathered}
y_{11}^{2} \leq 2 y_{1} y_{2}, \quad y_{12}^{2} \leq 2 y_{3} y_{4}, \quad y_{13}^{2} \leq 2 y_{5} y_{6}, \quad y_{14}^{2} \leq 2 y_{7} y_{8} \\
y_{21}^{2} \leq 2 y_{11} y_{12}, \quad y_{22}^{2} \leq 2 y_{13} y_{14} \\
x^{2} \leq 2 y_{21} y_{22}
\end{gathered}
$$

In the end, some constraints are redundant and we can only keep the following set of constraints :

$$
y_{11}^{2} \leq 2 x t, \quad y_{21}^{2} \leq 2 y_{11} y_{14}, \quad x^{2} \leq 2 y_{21} y_{22}, \quad y_{14}=2^{-11 / 2}, \quad y_{22}=2 s
$$

This set of constraint being equivalent to $x^{7 / 5} \leq s$, we have shown that it can be expressed using linear equality constraints and 3 rotated cone constraints of dimension 3 .

Therefore, the discrete problem for a Herschel-Bulkley model can also be modeled as a SOCP problem (obviously the number of auxiliary variables and rotated cone constraints will depend on the value of $m$ ).

\subsection{Comments}

Let us finish this section by a few remarks. First, it is to be noted that the introduction of auxiliary variables is only a way to express the problem in a standard SOCP form. In particular, there is no need to manage Lagrange multiplier fields or any penalization parameter. Obviously, the interior-point algorithms used to solve SOCP problems deal with constraints using penalization techniques (logarithmic barrier) but these have been optimized to tackle a certain class of problem, so that there is no need to tune any algorithmic input parameter, the choice of which being always a troublesome task.

The second remark concerns the scale and the structure of the problem. Let us consider, for instance, a problem discretized with 5000 elements, with a $n g=3$ Gauss point quadrature rule with a Bingham model. Then, there are 30000 cones, the number of optimization variables will be around 150000 and the number of linear constraints will be of the same order. Fortunately, the constraint matrix is sparse (its structure being roughly block-diagonal) so that the total number of non-zero elements will actually be much smaller than the size of the matrix of order $10^{5} \times 10^{5}$. However, 30000 conic constraints in $\mathbb{R}^{4}$ or $\mathbb{R}^{5}$ is still pretty large so that this problem can be considered as a large-scale problem. The interest of this method, as will be illustrated in the next sections, is that such a large-scale problem can still be solved very efficiently on a personal computer.

\section{Illustrative applications}

\subsection{Validation on the plane Poiseuille flow}

First, the plane Poiseuille flow of a Bingham and a Herschel-Bulkley fluid is considered and numerical velocity fields are compared to analytical solutions. 
Consider a rectangular fluid domain $\Omega=[0 ; L] \times[0 ; H]$ subjected to a uniform horizontal pressure-gradient $f=f \underline{e}_{x}$ with a no-slip boundary condition on the surfaces $y=0$ and $y=H$. Since there is no fluid motion in the $y$ direction for the exact Poiseuille flow, zero vertical velocities $u_{y}=0$ have been imposed on the surfaces $x=0$ and $x=L$ where the fluid flows in and out, for the numerical computations.

Let $u^{*}$ be the non-dimensional horizontal velocity such that the velocity field is given by $\underline{u}(x, y)=V u^{*}(y) \underline{e}_{x}$ where $V$ is the reference velocity. The analytical solution of this flow is given by the following relation :

$$
u^{*}(y)= \begin{cases}\frac{1}{M} f^{*}\left(\left(\frac{y_{0}}{H}\right)^{M}-\left(\frac{y-y_{0}}{H}\right)^{M}\right) & \text { if } 0 \leq y \leq y_{0} \\ \frac{1}{M} f^{*}\left(\left(\frac{y_{0}}{H}\right)^{M}-\left(\frac{y-\left(H-y_{0}\right)}{H}\right)^{M}\right) & \text { if } H-y_{0} \leq y \leq H \\ \frac{1}{M} f^{*}\left(\frac{y_{0}}{H}\right)^{M} & \text { if } y_{0} \leq y \leq H-y_{0}\end{cases}
$$

where $M=1+1 / m, f^{*}=(f H / K)^{1 / m} H / V$ is the non-dimensional pressure gradient and $y_{0}=H / 2-\tau_{0} / f=H / 2-B i H /\left(f^{*}\right)^{m}$ where $B i=\tau_{0} H^{m} / K V^{m}$ is the Bingham number. The velocity profile consists of two sheared liquid layers near the plate boundaries and a solid plug with a constant velocity in the central region. The width of the plug region being controlled by the Bingham number. Note that this solution is valid only if $B i / f^{*} \leq 1 / 2$, for $B i / f^{*}>1 / 2$ the fluid motion is completely stopped.

For numerical computations, we set $H=L=1, f^{*}=1$ whereas the (generalized) Bingham number has been varied. A structured mesh with 30 triangular elements in the $x$ direction and 10 in the $y$ direction $\left(N_{E}=600\right.$ in total) has been used for computations. It has been verified that the $y$-component of the velocity field was zero, and that the $x$ component was uniform with respect to the $x$ coordinate. Therefore, the horizontal velocity profile obtained at $x=L / 2$ has been compared to the analytical solution.

Both analytical and numerical profiles for different Bingham numbers have been represented in Figure 1 for the Bingham model $(m=1)$ and in Figure 2 for the Herschel-Bulkley $(m=0.4)$. Clearly, both profiles agree very well for all considered Bingham numbers. In particular, we observe that the fluid stops for $B i>0.5 f^{*}=0.5$. Note that an interesting feature of the proposed method is its ability to predict this critical pressure gradient. It can, indeed, be obtained by slightly changing the optimization problem in order to find the optimal loading factor corresponding to the onset of flow, as is classically done in yield design/limit analysis. This is a major advantage over regularizing models which are not able to predict such critical loadings.

\subsection{Lid-driven square cavity}

Another classical benchmark example is the lid-driven square cavity problem where noslip boundary conditions are applied to all edges except at the top surface which moves at 


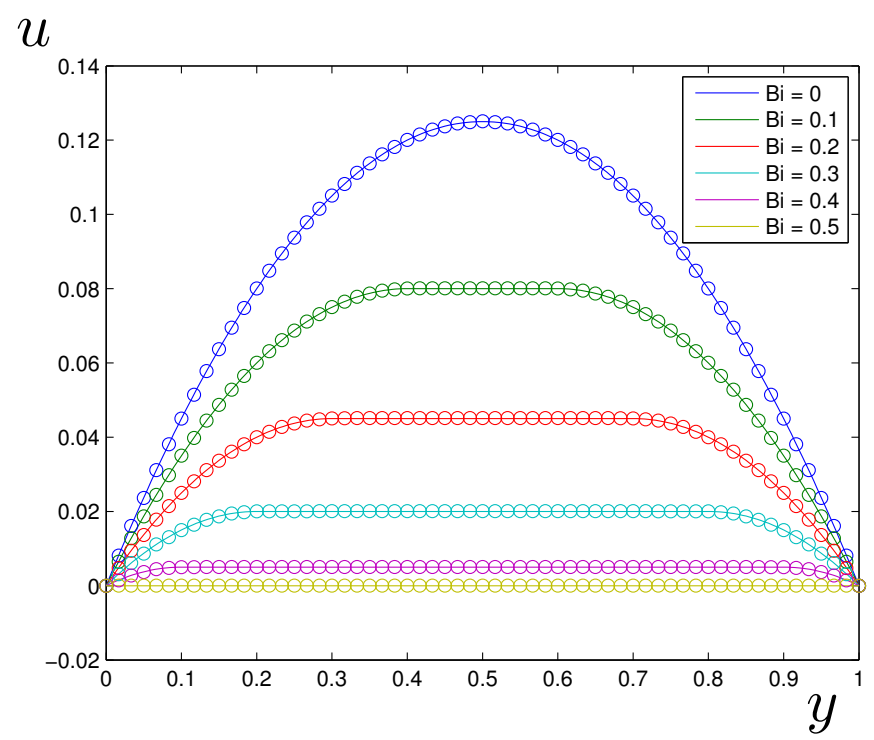

Figure 1: Evolution of the horizontal velocity profile of the plane Poiseuille flow for a Bingham fluid as a function of the Bingham number. $-=$ analytical solution, $\circ=$ numerical solution.

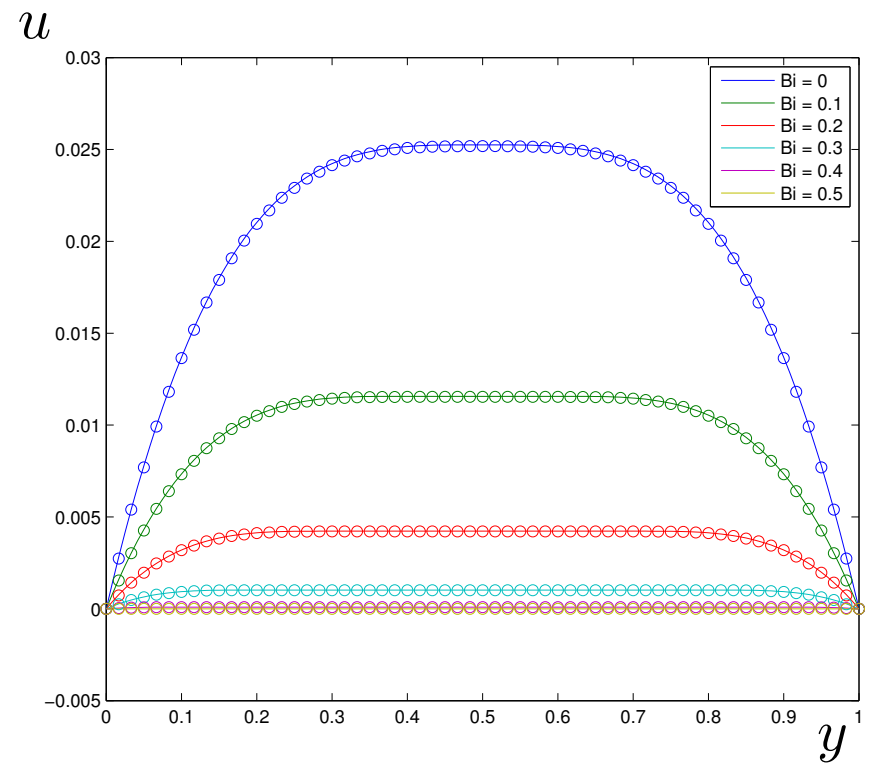

Figure 2: Evolution of the horizontal velocity profile of the plane Poiseuille flow for a Herschel-Bulkley fluid as a function of the Bingham number. - = analytical solution, o = numerical solution. 


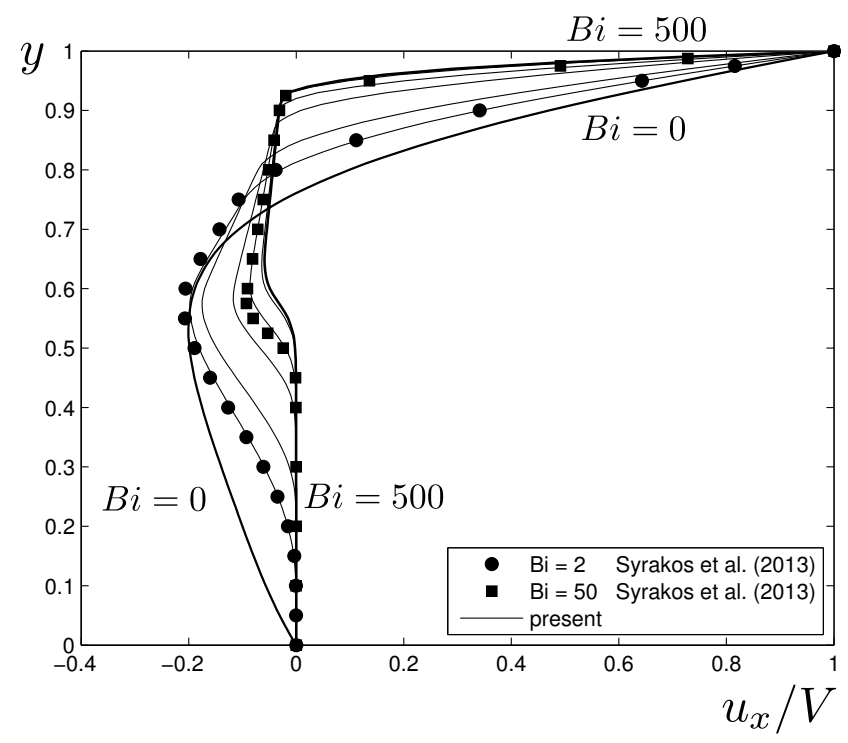

Figure 3: Horizontal velocity profiles along the axis $x=1 / 2$ and comparison to other simulations for $B i=2$ and $B i=50$. The Bingham numbers take the values $B i=0,2,5,20,50,200,500$, the two extremal thick lines correspond to $B i=0$ and $B i=500$.

a uniform horizontal velocity $V$.

A first computation on a mesh with 30 elements along each edges $(\Omega=[0 ; 1] \times[0 ; 1])$ has been performed for a Bingham fluid with different Bingham numbers $B i=0,2,5,20$, 50, 200, 500 and have been compared to the recent simulations of Syrakos for validation [4]. The different horizontal velocity profiles along the middle axis $x=1 / 2$ are represented in Figure 3. A very good agreement with the previous simulations is observed and the evolution of the velocity profile with respect to the Bingham number is consistent with results of [4]. In particular, it can be observed that the lower rigid region at rest is retrieved as well as the rigid region in the upper part which is animated by a rotating motion (linear part of the velocity profile) the angular velocity of which decreases with the Bingham number. The representation of the velocity fields inside the cavity (Figure 4) agree at least qualitatively with existing observations of the literature. It is to be noted that the fluid motions tends to a be localized in the vicinity of the driving lid as the Bingham number increases.

A mesh convergence study has been performed on these velocity profiles. The numerical solution obtained with a very fine mesh of $N_{E}=13474$ elements has been taken as the reference solution. The $L^{2}$ error between velocity profiles computed with 3 increasingly finer meshes and the reference solution has been computed for the considered Bingham numbers and reported in Table 1. It can be observed that, at a fixed Bingham number, the error decreases when refining the mesh. One can also notice the fact that for mesh 2 and 3 the error increases with the Bingham number. Besides, it has also been observed that total computation times increased with the Bingham number (by almost $50 \%$ between $B i=0$ 


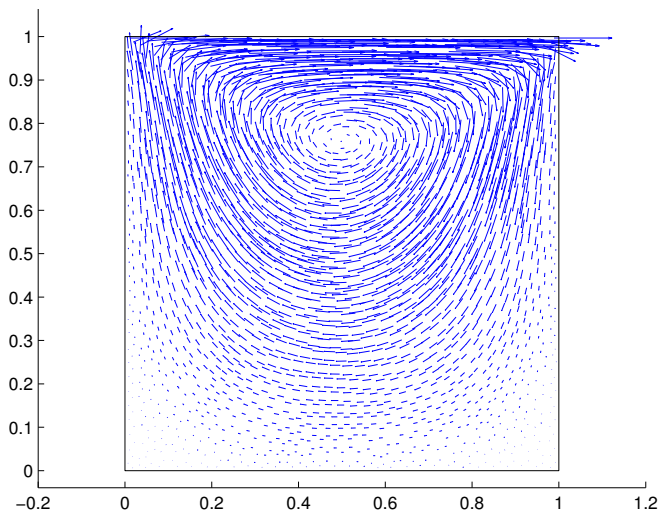

(a) $B i=0$

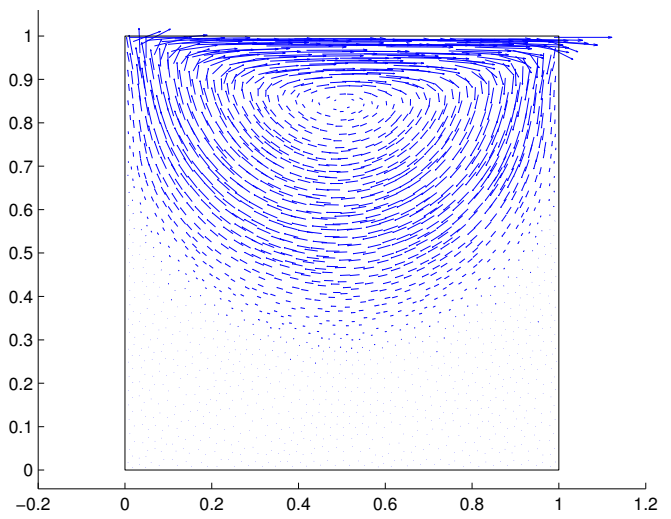

(c) $B i=5$

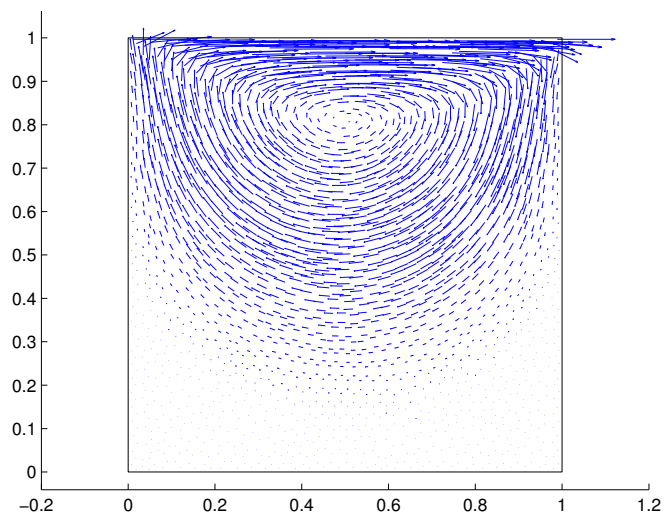

(b) $B i=2$

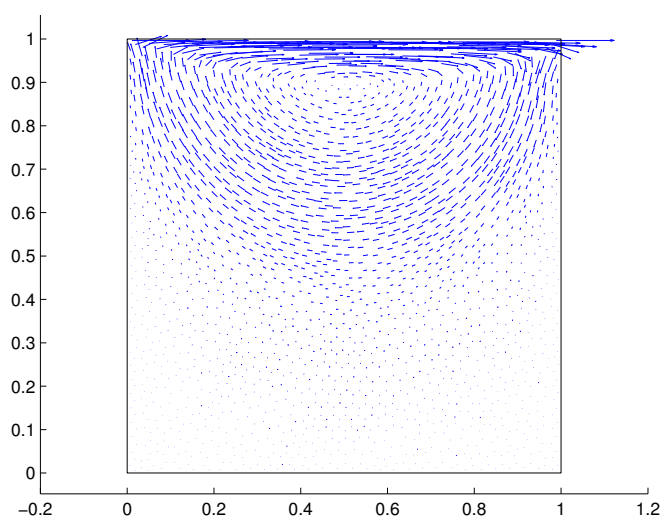

(d) $B i=50$

Figure 4: Velocity fields of the lid-driven cavity problem

\begin{tabular}{cccccccc}
\hline$B i$ & 0 & 2 & 5 & 20 & 50 & 200 & 500 \\
\hline $\begin{array}{c}\text { Mesh 1 } \\
\left(N_{E}=252\right)\end{array}$ & 0.0040 & 0.0052 & 0.1325 & 0.1591 & 0.0849 & 0.1022 & 0.1190 \\
\hline $\begin{array}{c}\text { Mesh 2 } \\
\left(N_{E}=2084\right)\end{array}$ & 0.0005 & 0.0019 & 0.0036 & 0.0073 & 0.0121 & 0.0305 & 0.0446 \\
\hline $\begin{array}{c}\text { Mesh 3 } \\
\left(N_{E}=5798\right)\end{array}$ & 0.0005 & 0.0009 & 0.0028 & 0.0043 & 0.0052 & 0.0066 & 0.0137 \\
\hline
\end{tabular}

Table 1: Evolution of the $L^{2}$ error on the velocity profile at $x=1 / 2$ with respect to a reference numerical solution for increasingly finer meshes. 
Mesh 1 Mesh 2 Mesh 3

\begin{tabular}{cccc}
\hline nb. of elements $N_{E}$ & 252 & 2084 & 5798 \\
\hline linear constraints & 6918 & 56622 & 157140 \\
conic constraints & 1512 & 12504 & 34788 \\
optimization variables & 8316 & 68772 & 191334 \\
\hline nb. of iterations & 23 & 32 & 39 \\
reordering time $(\mathrm{s})$ & 0.39 & 4.3 & 13.7 \\
optimization time $(\mathrm{s})$ & 0.46 & 6.2 & 12.9 \\
total time $(\mathrm{s})$ & 0.85 & 10.5 & 36.6 \\
\hline
\end{tabular}

Table 2: Computation statistics for different meshes with a Bingham fluid $(B i=500)$

Mesh 1 Mesh 2 Mesh 3

\begin{tabular}{cccc}
\hline nb. of elements $N_{E}$ & 252 & 2084 & 5798 \\
\hline linear constraints & 9942 & 81630 & 226716 \\
conic constraints & 3024 & 25008 & 69576 \\
optimization variables & 12852 & 106284 & 295698 \\
\hline nb. of iterations & 23 & 31 & 38 \\
presolve time (s) & 0.17 & 7.3 & 18 \\
optimization time (s) & 0.16 & 5.8 & 27.2 \\
total time (s) & 0.33 & 13.1 & 45.2 \\
\hline
\end{tabular}

Table 3: Computation statistics for different meshes with a HB fluid $(B i=500)$

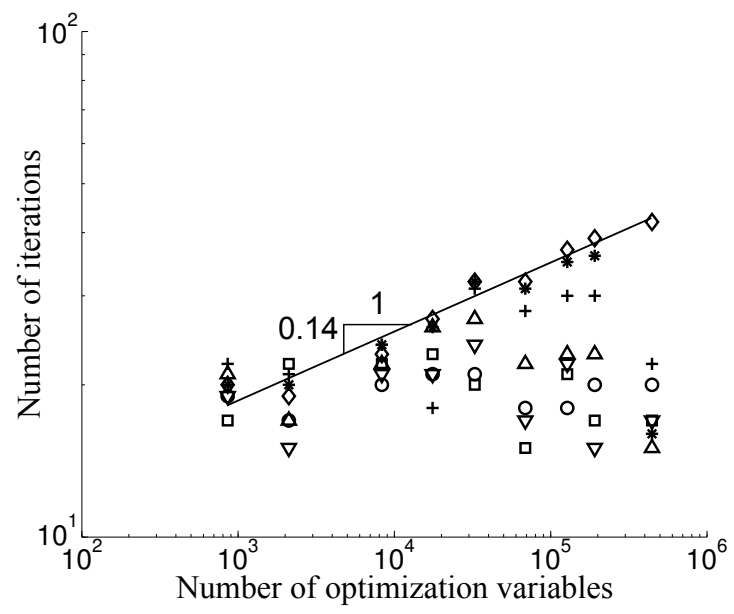

(a) Bingham model

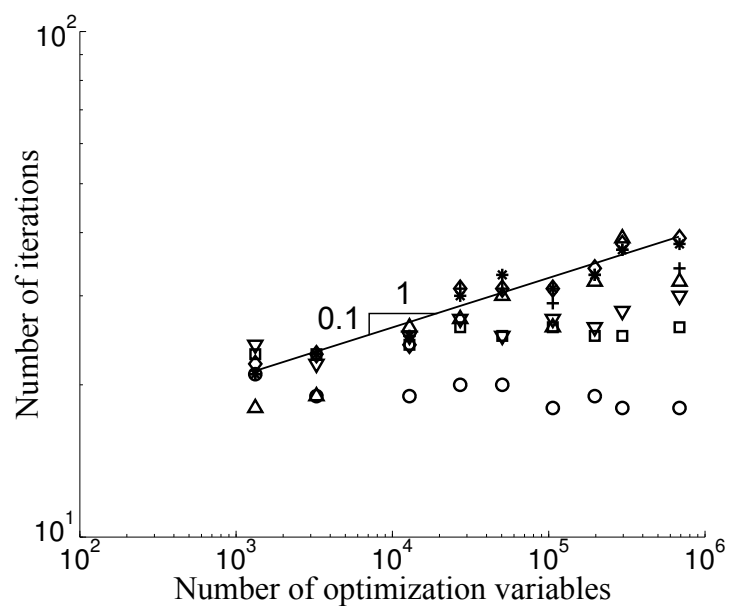

(b) Herschel-Bulkley model

Figure 5: Dependance of the number of iterations with respect to the total number of optimization variables. $\bigcirc: B i=0, \square: B i=2, \nabla: B i=5, \triangle: B i=20,+: B i=50, *: B i=200, \diamond: B i=500$ 


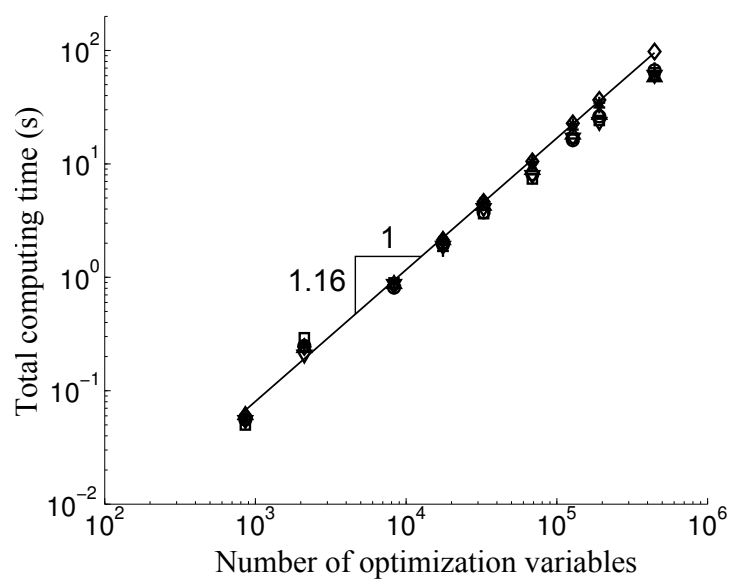

(a) Bingham model

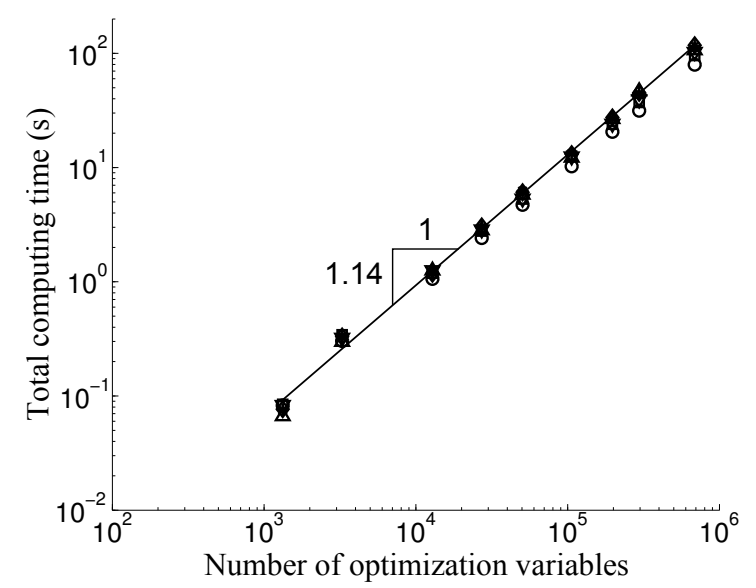

(b) Herschel-Bulkley model

Figure 6: Dependance of the total computing time with respect to the total number of optimization variables. $\bigcirc: B i=0, \square: B i=2, \nabla: B i=5, \triangle: B i=20,+: B i=50, *: B i=200, \diamond: B i=500$

and $B i=500)$. This can be easily explained because, for high Bingham numbers, the solution is dominated by yield effects which are the cause of the numerical difficulties due to the non-smoothness of the yield term. However, it is important to note that the present method remains perfectly suitable for treating the case of zero viscosity or infinite Bingham numbers.

Finally, some statistics in terms of size and computing times of the optimization problems which have been solved are presented in Table 2 for $B i=500$. For the three considered meshes, the number of optimization variables, linear constraints and conic constraints are given. As regards computing times, the reordering time corresponds to a procedure of MoseK used to reorder the linear equality constraints. Therefore, the total computing time includes the preordering time and the time needed for the actual optimization part. The time needed to assemble the matrices used to formulate the problem has not been taken into account since it is not very important and can be optimized using an appropriate programming language. Note that all computations have been performed on a Intel-P4 2.4 $\mathrm{GHz}$ running Linux 32-bits using MoseK v7.0, finite element meshes have been generated using GMSH and the matrices have been assembled in MatLaB 7.11 (R2010b). It is to be observed that the total computing time for a relatively large problem (mesh 3 with a few hundred of thousands of variables) remains moderate for both models.

Such performances are further illustrated by figure 5 representing the number of iterations of the interior point solver as a function of the total number of optimization variables. One can observe that, even for a large range of optimization variables, the number of iterations exhibits a very weak dependency with respect to the problem size. This is a particular feature of such algorithms and also illustrates that the practical complexity of the number of iterations required to converge is, in general, better than the theoretical one which predicts a complexity of $O\left(\sqrt{N} \log \frac{1}{\epsilon}\right)$ where $N$ is the number of variables and $\epsilon$ the desired 


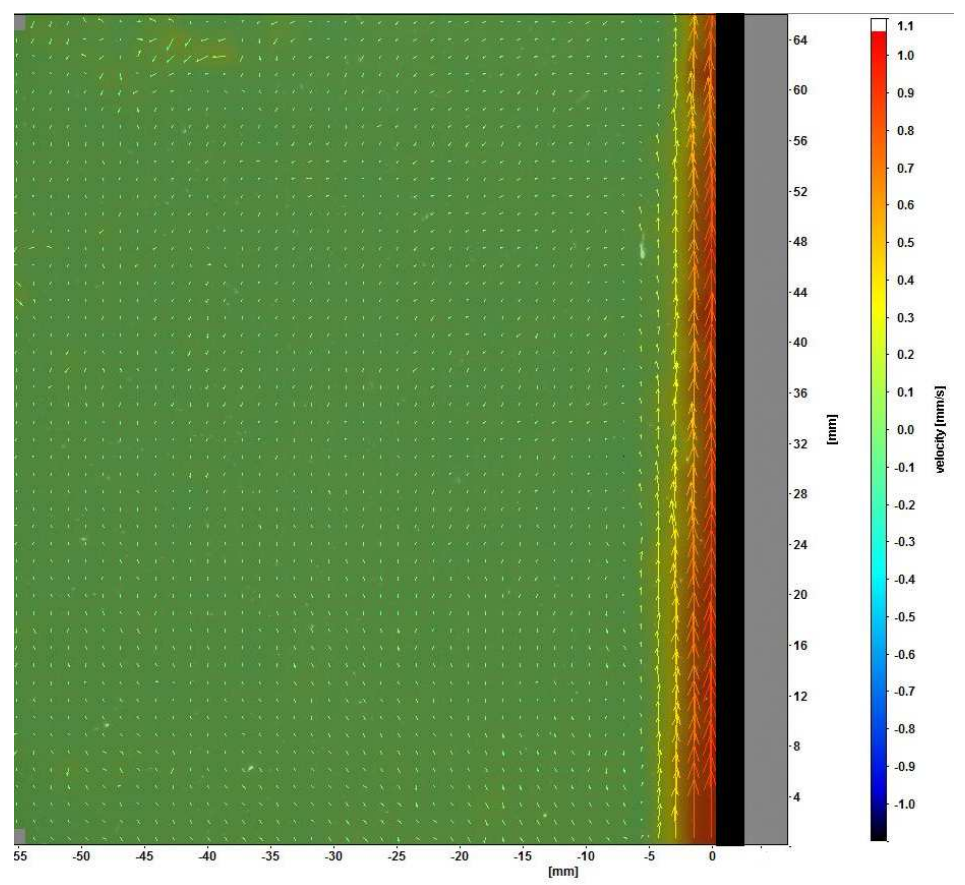

(a) Experimental velocity field obtained by PIV in a region close to the plate $\left(V=1 \mathrm{~mm} / \mathrm{s}, \tau_{0}=34 \mathrm{~Pa}, K=13.9 \mathrm{UI}, m=0.35\right)$

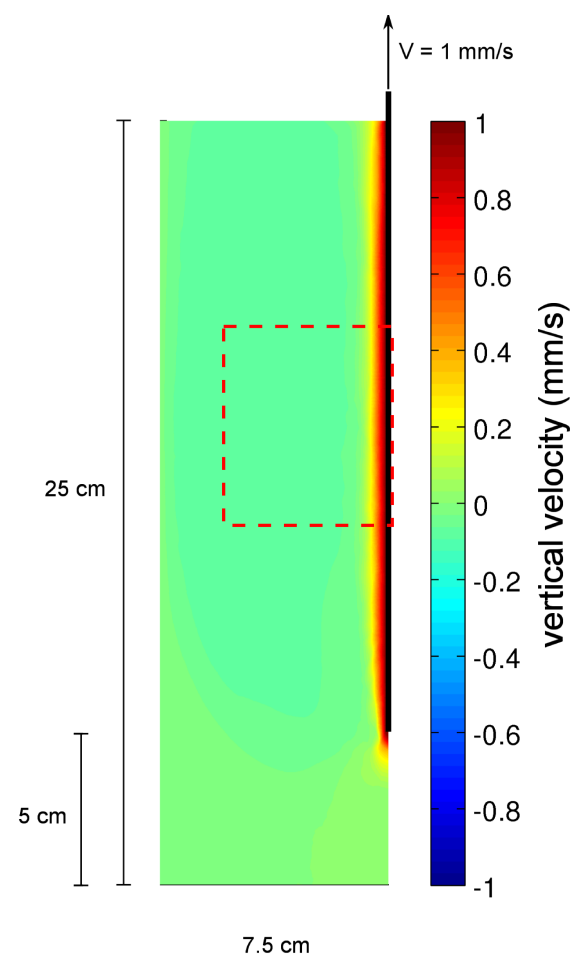

(b) Velocity field obtained by numerical computation in the fluid bath $(V=1$ $\mathrm{mm} / \mathrm{s}$ ) and corresponding window of observation for the experimental measures

Figure 7: Comparison of velocity fields

accuracy [29]. In particular for the highest Bingham numbers, we see here that the number of iteration complexity is roughly in $O\left(N^{1 / 10}\right)-O\left(N^{1 / 6}\right)$ for a fixed accuracy. Besides, due to the high sparsity of the constraint matrix, each iteration can be solved with a very good time complexity (almost linear), so that the complexity of the total computing time is almost linear $\left(O\left(N^{1.15}\right)\right)$ as illustrated in figure 6 . This remark confirms the fact that such algorithms present, in practice, a low-order polynomial time complexity and this seem to be independent from the viscosity model (Bingham or Herschel-Bulkley).

Unfortunately, we were not able to find references of computing times using traditional techniques such as the Augmented Lagrangian in the available literature, but, to the authors opinion, such computing times may probably be much larger since the Augmented Lagrangian presents, in general, a much stronger dependance of the number of iterations with respect to the problem size. 


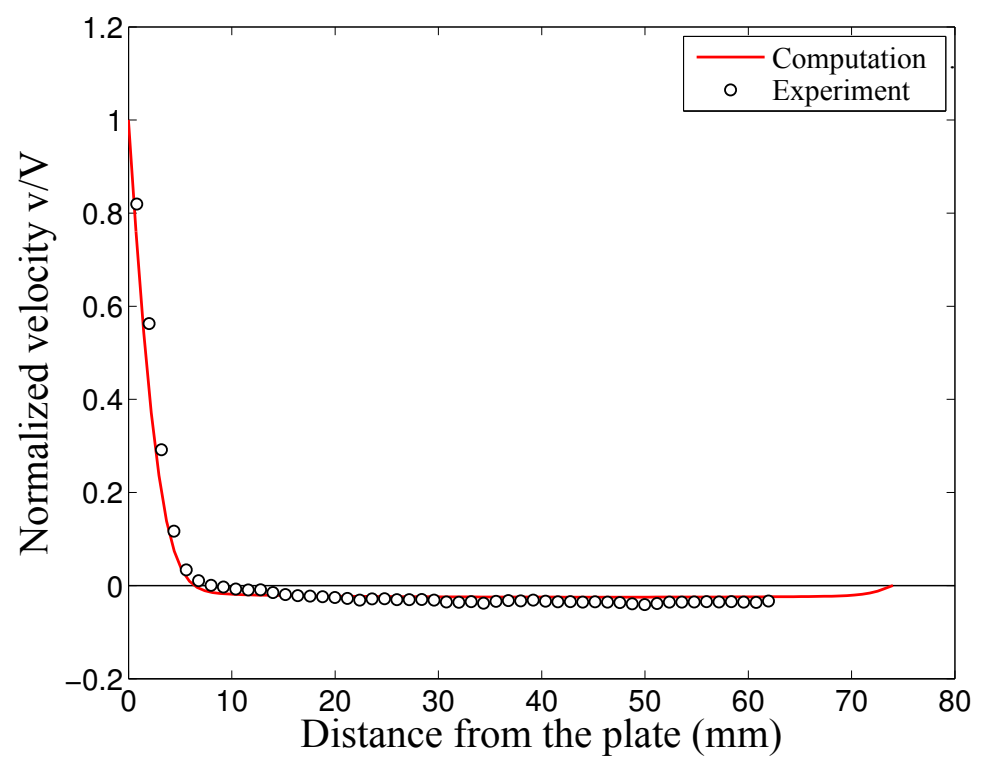

Figure 8: Vertical velocity profile in the plate central zone $(V=0.5 \mathrm{~mm} / \mathrm{s})$

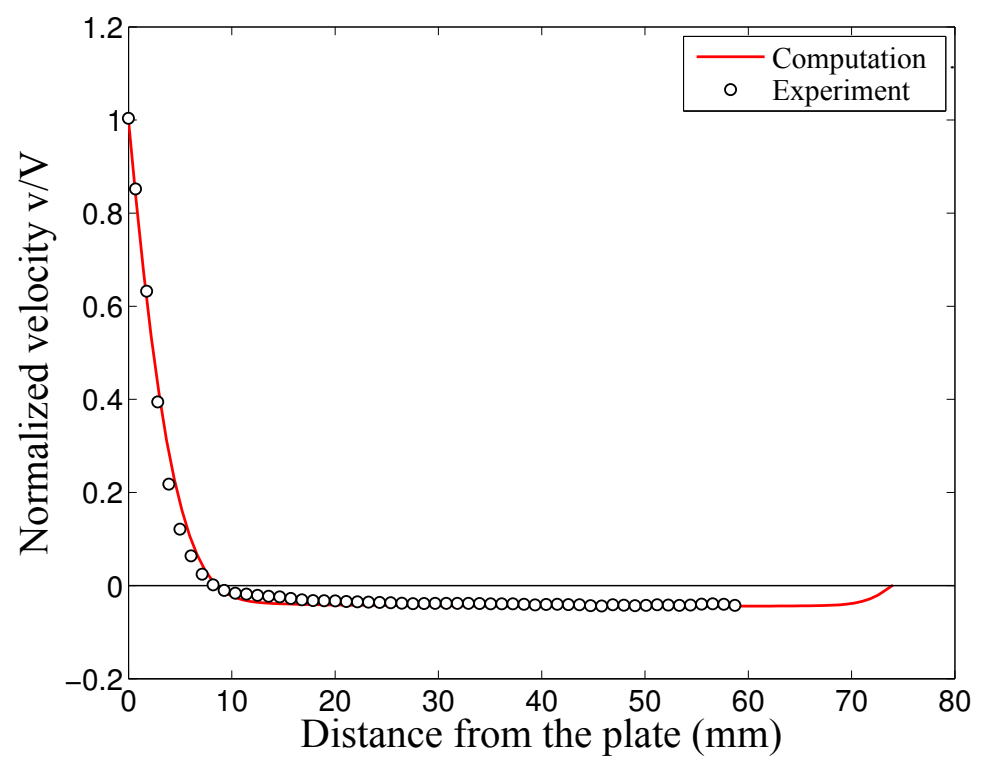

Figure 9: Vertical velocity profile in the plate central zone $(V=5 \mathrm{~mm} / \mathrm{s})$ 


\subsection{Flow of a $H B$ fluid around a moving plate}

The proposed method is finally validated by modeling the flow generated by the displacement of a partially immersed plate in a bath of yield stress fluid [30, 31]. In the experiments, the plate is withdrawn at constant velocity in the range $\left[0.2 ; 17 \mathrm{~mm} . \mathrm{s}^{-1}\right]$ from a bath of Carbopol gel following a HB behavior with $\tau_{0}=34 \mathrm{~Pa}, K=13.9 \mathrm{~Pa} . \mathrm{s}^{-m}$ and $m=0.35$. The fluid domain is a $25 \mathrm{~cm}$-height, $10 \mathrm{~cm}$-width and $15 \mathrm{~cm}$-length parallelepiped, the plate being covered with sandpaper in order to prevent any slippage. Its thickness is $1.88 \mathrm{~mm}$, its height $25 \mathrm{~cm}$ and its width $7 \mathrm{~cm}$. The velocity field around the plate is determined in a plane perpendicular to the plate, along its central axis using the Particle Image Velocimetry (PIV) technique.

Due to the problem symmetry, only half of the fluid bath has been modeled. A perfect adherence to the walls and the plate has been assumed and the fluid surface is stress-free. A vertical velocity of intensity $V$ has been imposed to the plate. The computed velocity fields are compared to the velocities observed once a steady state has been established in the fluid bath.

Figure 7(a) shows the experimental vertical velocity field around the plate far from the free surface and the plate tip while Figure $7(\mathrm{~b})$ presents the numerical velocity field computed with the same parameters (except with $m=0.4$ ). In both cases the fluid is strongly sheared upwards in a small layer along the plate, whereas it slightly moves downwards far from the plate. Besides, the velocity profile is uniform along the plate in a region away from the free surface and the plate tip. More precisely, the vertical velocity profiles in this region have been represented as a function of the distance from the plate and are compared against experimental values in figures 8 and 9 . The simulations correctly reproduced the specific features of this flow: there is a linear velocity gradient near the plate and a negative velocity plateau in the fluid recirculation area.

\section{Conclusions}

A new optimization technique to solve minimization problems arising in the simulation of yield stress fluid flows has been presented. The method relies on a standard finite element discretization, on the formulation of the discretized minimization problem as a second-order cone program and on the use of dedicated efficient interior-point solvers, like the industrial software package MoseK. The advantage of using such techniques over traditional approaches, like the Augmented Lagrangian for instance, have been underlined and computing times as functions of the size of the problem have been reported on a benchmark example. An excellent time complexity (almost linear) has been observed for the Bingham as well as for the Herschel-Bulkley model and for a wide range of Bingham numbers. The method has been further validated on the simulation of the flow generated by the plate displacement in a bath of yield stress fluid by comparing the obtained velocity fields to experimental measurements.

It is important to note that the proposed approach can be easily generalized to other types of problems. Indeed, for the sake of simplicity, the present work has been restricted to 
a 2D plane strain steady state setting. The generalization to axisymmetric or 3D problems will affect only the finite element discretization process whereas the problem formulation as a second-order cone program will have exactly the same structure. Similarly, the simulation of transient flows can be also modeled using the proposed approach as it requires the resolution of similar problems at each time step. To the author's opinion, the simulation of transient 3D flows in complex geometry would greatly benefit from the computational efficiency of the proposed method.

\section{References}

[1] M. Bercovier, M. Engelman, Journal of Computational Physics 36 (1980) 313-326.

[2] T. C. Papanastasiou, Journal of Rheology 31 (1987) 385.

[3] D. D. dos Santos, S. Frey, M. F. Naccache, P. R. de Souza Mendes, Journal of Non-Newtonian Fluid Mechanics 166 (2011) 667-679.

[4] A. Syrakos, G. C. Georgiou, A. N. Alexandrou, Journal of Non-Newtonian Fluid Mechanics 195 (2013) $19-31$.

[5] M. Fortin, R. Glowinski, Méthodes de lagrangien augmenté: applications à la résolution numérique de problèmes aux limites, Dunod, 1982.

[6] P. Saramito, N. Roquet, Computer methods in applied mechanics and engineering 190 (2001) 53915412.

[7] J. Zhang, Computer Methods in Applied Mechanics and Engineering 199 (2010) 3051-3057.

[8] E. J. Dean, R. Glowinski, G. Guidoboni, Journal of non-newtonian fluid mechanics 142 (2007) 36-62.

[9] J. Salençon, Calcul à la rupture et analyse limite, Presses de l'Ecole Nationale des Ponts et Chaussées, 1983.

[10] J. Salençon, Yield Design, Wiley. com, 2013.

[11] A. Friaâ, CR Acad. Sci. Paris Sér. AB 286 (1978) A953-A956.

[12] T. Guennouni, P. Le Tallec, J. Méca. Théo. Appl. Mech 2 (1982) 75-99.

[13] J. Pastor, S. Turgeman, J. de Mécanique Théorique et Appliquée 2 (1983) 393-416.

[14] S. Turgeman, J. Pastor, Journal de mécanique théorique et appliquée 6 (1987) 121-143.

[15] S. W. Sloan, International Journal for Numerical and Analytical Methods in Geomechanics 12 (1988) $61-77$.

[16] S. W. Sloan, International Journal for Numerical and Analytical Methods in Geomechanics 13 (1989) 263-282.

[17] K. D. Andersen, E. Christiansen, M. L. Overton, SIAM Journal on Scientific Computing 19 (1998) $1046-1062$.

[18] A. V. Lyamin, S. W. Sloan, International Journal for Numerical Methods in Engineering 55 (2002) 573-611.

[19] A. V. Lyamin, S. W. Sloan, International Journal for Numerical and Analytical Methods in Geomechanics 26 (2002) 181-216.

[20] Mosek, The Mosek optimization toolbox for Matlab manual, 2008.

[21] A. Makrodimopoulos, International Journal for Numerical Methods in Biomedical Engineering 26 (2010) $1449-1461$.

[22] A. Makrodimopoulos, C. M. Martin, International Journal for Numerical and Analytical Methods in Geomechanics 31 (2007) 835-865.

[23] H. Ciria, J. Peraire, J. Bonet, International Journal for Numerical Methods in Engineering 75 (2008) 899-944.

[24] M. A. A. Skordeli, C. D. Bisbos, Engineering Structures 32 (2010) 1556-1567.

[25] C. V. Le, H. Nguyen-Xuan, H. Nguyen-Dang, Computers \& Structures 88 (2010) 65-73.

[26] J. Bleyer, P. de Buhan, International Journal for Numerical Methods in Engineering 94 (2013) 308-330. 
[27] P. Saramito, Méthodes numériques en fluides complexes : théorie et algorithmes, 2013. Cours de DEA, Laboratoire Jean Kuntzmann (LJK)-Grenoble.

[28] A. Ben-Tal, A. S. Nemirovskiı̌, Lectures on modern convex optimization: analysis, algorithms, and engineering applications, Siam, 2001.

[29] J. Gondzio, European Journal of Operational Research 218 (2012) 587-601.

[30] J. Boujlel, M. Maillard, A. Lindner, G. Ovarlez, X. Chateau, P. Coussot, Journal of Rheology 56 (2012) $1083-1108$

[31] M. Maillard, J. Boujlel, P. Coussot, Physical review letters 112 (2014) 068304. 